\title{
Editorial: Prognostic evaluation of retinal detachment
}

In attempting to review the numerous inter-related factors that must be considered in the assessment of prognosis of retinal detachment, rigid and absolute criteria cannot be established. Instead each case requires individual evaluation based on the pertinent ocular findings, family history, and possibly, biochemical analysis of subretinal fluid.

Knowledge of the expected progression of a retinal detachment in relation to a retinal break enables the surgeon to assess the likely danger to the macula, a matter of primary concern in functional prognosis. Once the macula becomes detached, regardless of how quickly it is reapposed, there will inevitably be some loss of central vision. Lincoff (196I) has shown that subretinal fluid tends to orientate itself dependently and symmetrically in a predictable manner around the retinal break responsible for its occurence. For this reason retinal detachments due to breaks situated in the superotemporal quadrant are most dangerous as macular involvement is likely to occur at an early stage; conversely, detachments due to inferonasal breaks progress slowly and any possible macular involvement occurs relatively late.

Retinal detachments associated with posteriorly located breaks, such as macular holes, and those occurring in some cases of diabetic traction detachments, usually carry an unfavourable prognosis. This is due to the relative inaccessibility of the sclera posterior to the equator which makes scleral buckling procedures in this region technically difficult and traumatic. Other unfavourable prognostic characteristics are retinal breaks that extend for more than one quadrant of the circumference of the globe (Schepens and Freeman, 1967), and those detachments that occur after surgery for congenital cataracts (Kanski, Elkington, and Daniel, 1974).

The presence of massive vitreous retraction (MVR) has a profound influence on prognosis. In MVR the retina becomes immobilized by fibrous membranes so that conventional surgery usually fails to reappose the retina permanently. In its early or pre-fibrotic stage, the peripheral retina becomes immobilized, retinal breaks become distorted with rolled posterior edges, and the vitreous becomes less mobile so that it appears to move as a whole in one inelastic mass with no flow after the cessation of ocular movements. An essential feature of MVR is the presence within the vitreous of large pigment cells which are thought to be directly responsible for the formation of epiretinal membranes. In its advanced or fibrotic stage, the retina is invariably totally detached and funnelled, with puckering and narrowing of posterior retinal folds. Membranes in the retina and vitreous become visible on slit-lamp examination, and there is an increase in the amount of pigment cells in the vitreous. Once the fibrotic stage has developed conventional surgery has no chance of success (Scott, 1973).

Also meriting attention in an evaluation of prognosis is the presence of a family history of retinal detachment. A number of studies have demonstrated that heredo-familial occurrence of retinal detachment associated with high degrees of myopia, vitreous degeneration, retinoschisis, severe lattice degeneration of the retina, and general disorders such as Marfan's syndrome, and clefting syndrome, are commonly associated with a relatively poor prognosis.

The article appearing on p. 70 shows that the biochemical analysis of subretinal fluid can be used as a prognostic indicator. The authors found that low levels of total protein, sialic acid, and hexosamides in the subretinal fluid were characteristic of patients with recent retinal detachments and a favourable prognosis.

\section{References}

LINCOFf, H. A. (196I) Arch. Ophthal. (Chicago), 66, 48 schepens, c. L., and freeman, H. м. (1967) Trans. Amer. Acad. Ophthal. Otolaryng., 71, 474

KANSKI, J. J., Elkington, A. E., and DANIEL, R. (1974) Brit. J. Ophthal., 58, 92

scoTT, J. D. (1973) Trans. ophthal. Soc. U.K., 93, 417 